\title{
Early Outcomes of Robotic Versus Video-Assisted Thoracoscopic Anatomical Resection for Lung Cancer
}

\author{
Ji Hyeon Park, M.D., Samina Park, M.D., Chang Hyun Kang, M.D., Ph.D., Bub Se Na, M.D., So Young Bae, M.D., \\ Kwon Joong Na, M.D., Hyun Joo Lee, M.D., In Kyu Park, M.D., Ph.D., Young Tae Kim, M.D., Ph.D. \\ Department of Thoracic and Cardiovascular Surgery, Seoul National University Hospital, Seoul National University College of Medicine, Seoul, Korea
}

\author{
ARTICLE INFO \\ Received October 14, 2021 \\ Revised November 23, 2021 \\ Accepted November 26, 2021 \\ Corresponding author \\ Chang Hyun Kang \\ Tel 82-2-2072-3010 \\ Fax 82-2-762-3566 \\ E-mail chkang@snu.ac.kr \\ ORCID \\ https://orcid.org/0000-0002-1612-1937
}

\begin{abstract}
Background: We compared the safety and effectiveness of robotic anatomical resection and video-assisted thoracoscopic surgery (VATS).

Methods: A retrospective analysis was conducted of the records of 4,283 patients, in whom an attempt was made to perform minimally invasive anatomical resection for lung cancer at Seoul National University Hospital from January 2011 to July 2020. Of these patients, 138 underwent robotic surgery and 4,145 underwent VATS. Perioperative outcomes were compared after propensity score matching including age, sex, height, weight, pulmonary function, smoking status, performance status, comorbidities, type of resection, combined bronchoplasty/angioplasty, tumor size, clinical T/N category, histology, and neoadjuvant treatment.

Results: In total, 137 well-balanced pairs were obtained. There were no cases of 30-day mortality in the entire cohort. Conversion to thoracotomy was required more frequently in the VATS group (VATS $6.6 \%$ vs. robotic $0.7 \%, p=0.008$ ). The complete resection rate (VATS $97.8 \%$ vs. robotic $98.5 \%, p=1.000$ ) and postoperative complication rate (VATS $17.5 \%$ vs. robotic $19.0 \%, p=0.874$ ) were not significantly different between the 2 groups. The robotic group showed a slightly shorter hospital stay (VATS 5.8 \pm 3.9 days vs. robotic 5.0 \pm 3.6 days, $\mathrm{p}=0.052$ ). N2 nodal upstaging ( $\mathrm{cN} 0 / \mathrm{pN} 2$ ) was more common in the robotic group than the VATS group, but without statistical significance (VATS 4\% vs. robotic $12 \%, p=0.077$ ).

Conclusion: Robotic anatomical resection in lung cancer showed comparable early outcomes when compared to VATS. In particular, robotic resection presented a lower conversion-to-thoracotomy rate. Furthermore, a robotic approach might improve lymph node harvesting in the N2 station.
\end{abstract}

Keywords: Robotic surgical procedures, Lung neoplasms, Minimally invasive surgery

\section{Introduction}

Minimally invasive surgery has become a standard technique for lung cancer worldwide. Video-assisted thoracoscopic surgery (VATS) has been established as the gold standard for early-stage lung cancer based on its superiority to open thoracotomy in terms of low postoperative morbidity and comparable long-term survival [1,2]. Meanwhile, robot-assisted thoracoscopic surgery (RATS) has recently gained popularity due to its improved vision and the advantages offered by robotic instruments. Technical advances in robotic systems have provided a less invasive method with oncologic completeness for the surgical treatment of lung cancer.

Previous studies showed comparable short-term and long-term outcomes in RATS for lung cancer compared to VATS and thoracotomy [3-6]. Most of all, adequate lymph node yields have been confirmed $[7,8]$. Furthermore, RATS has been shown to be feasible and safe even in cases of locally advanced lung cancer and in technically demanding procedures such as sleeve resection or angioplasty, where RATS can achieve oncologic integrity [9-11].

Hence, we evaluated the safety and effectiveness of robotic anatomical resection in comparison to VATS at a high-volume center. 


\section{Methods}

\section{Ethical statement}

The study was approved by the Institutional Review Board of Seoul National University Hospital. The requirement for individual consent was waived (approval no., H-2010-114-1165).

\section{Patients}

We conducted a retrospective study of patients who underwent curative anatomical resection via VATS or RATS for lung cancer between January 2011 and July 2020. We excluded patients who underwent (1) intentional thoracotomy, (2) non-curative surgery (i.e., biopsy only), and (3) non-anatomical resection. A total of 4,283 patients were enrolled in this study, including 4,145 who underwent VATS and 138 who underwent RATS (Fig. 1).

There were no differences in the preoperative and postoperative treatment protocol between the approaches. Data on patients' baseline characteristics, pathologic results, and short-term outcomes were collected. The postoperative complications were described according to the ClavienDindo classification. The clinical and pathologic stages were evaluated according to the seventh edition of the TNM classification for lung cancer.

\section{Operative techniques}

VATS has been adopted for lung cancer since 2005, and currently over $80 \%$ of patients with lung cancer undergo curative resection via VATS at our institution. We implemented RATS for lung cancer in 2011 using the da Vinci system (Intuitive, Sunnyvale, CA, USA). After our early experience, RATS has been performed based on conventional port placement following previous research $[12,13]$. However, this technique has some drawbacks associated with the need for an assistant surgeon and a small working space in the Asian population. Moreover, since the da Vinci Xi system was released, robotic staplers can be fully utilized in thoracic surgery. Therefore, we have modified robotic port placement for lung cancer, with an anterolateral approach [14]. We believe that the current port placement maximizes the advantages of robotic surgery, which are as follows: (1) full use of the 4 robotic arms, (2) solo surgery without the need of an assistant's help, (3) sufficient distance between the robotic arms in patients with small chests, and (4) sufficient intrathoracic space for a robotic stapler. The first utility incision was made in the fifth intercostal space along the anterior axillary line, measuring 3 $\mathrm{cm}$. In patients requiring tissue diagnosis before anatomic lung resection, wedge resection was usually performed by an uniportal technique through this utility incision. The second port was made $7.5 \mathrm{~cm}$ below the first port and was usually located in the seventh intercostal space along the anterior axillary line. The third port was the camera port, which was made in the ninth intercostal space along the midaxillary line. The fourth port was made in the 11th intercostal space, along the posterior axillary line, anterior to the scapular tip since a posterior location can increase the conflict between the grasper and the vertebral body during subcarinal lymph node dissection [14].

\section{Statistical analysis}

All statistical analyses were performed using IBM SPSS ver. 22.0 (IBM Corp., Armonk, NY, USA). The Student t-test and Wilcoxon rank-sum test were used to compare continuous variables. Propensity score matching was used

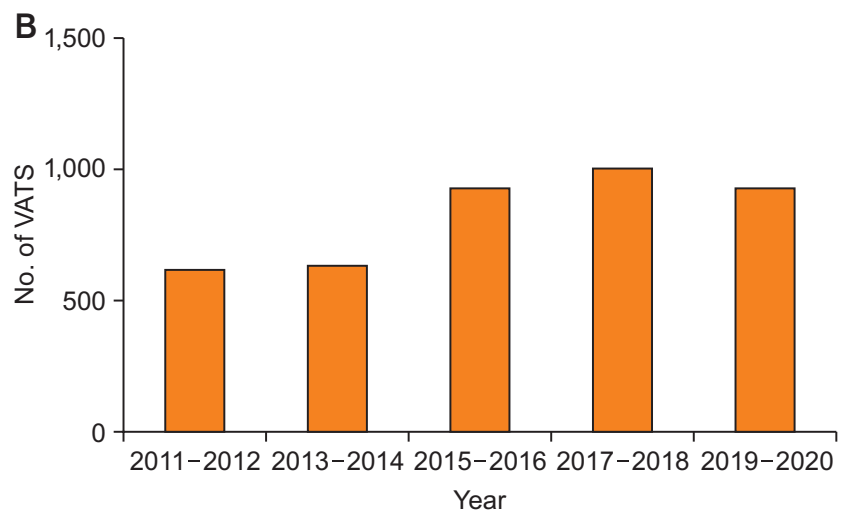

Fig. 1. Number of operations per year: (A) robot-assisted thoracoscopic surgery (RATS) and (B) video-assisted thoracoscopic surgery (VATS). 
to adjust for between-group differences in baseline characteristics and disease severity. The propensity scores were based on logistic regression modeling, which included the following variables: age, sex, height, weight, pulmonary function, smoking status, performance status, comorbidities, type of resection, combined bronchoplasty/angioplasty, tumor size, clinical T/N category, histology and neoadjuvant treatment. We matched the propensity scores 1-to-1 using the nearest-neighbor method without replacement, with a 0.2 caliper width. After the matching procedure, 137 patients from each group were selected for analysis. All statistical analyses were performed using 2 -sided tests, and statistical significance was set at $\mathrm{p}<0.05$.

\section{Results}

The baseline characteristics of the study population before and after matching are shown in Table 1. The distribution of age, sex, height, weight, pulmonary function, smoking status, performance status, comorbidities, type of resection, combined bronchoplasty/angioplasty, tumor size, clinical T/N category, histology, and neoadjuvant treatment were comparable between the 2 groups after propensity score matching. In the matched cohort, the between-group difference in terms of whether bronchoplasty/angioplasty was performed decreased (VATS 5.1\% versus RATS 6.6\%). Most patients enrolled in this study were in clinical stage T1 (VATS 70.8\% versus RATS 62.0\%) and N0 (VATS 94.2\% versus RATS $89.8 \%$ ). The neoadjuvant treatment rate was $0.7 \%$ and $1.5 \%$ in VATS and RATS, respectively. Most of the patients in the study underwent mediastinal lymph node dissection, and fewer than $3 \%$ of patients underwent mediastinal lymph node sampling.

\section{Operative outcomes}

Table 2 demonstrates the operative outcomes. In the matched cohort, there were no cases of 30-day mortality in the entire cohort. Conversion to thoracotomy was significantly lower in the RATS group than in the VATS group (VATS 6.6\% versus RATS $0.7 \%, \mathrm{p}=0.008)$. Nine cases $(6.6 \%)$ in the matched VATS group required conversion to thora-

Table 1. Preoperative characteristics of the study population

\begin{tabular}{|c|c|c|c|c|c|c|}
\hline \multirow{2}{*}{ Characteristic } & \multicolumn{3}{|c|}{ Unmatched patients } & \multicolumn{3}{|c|}{ Propensity score-matched patients } \\
\hline & VATS $(\mathrm{N}=4,145)$ & RATS ( $N=138)$ & SMD & VATS $(\mathrm{N}=137)$ & RATS (N=137) & SMD \\
\hline Age $(y r)$ & $63.4 \pm 9.8$ & $63.9 \pm 9.6$ & -0.02 & $63.4 \pm 10.7$ & $63.9 \pm 9.7$ & 0.06 \\
\hline Sex (male) & $2,129(51.4)$ & $72(52.2)$ & 0.02 & $64(46.7)$ & $71(51.8)$ & 0.10 \\
\hline Height $(\mathrm{cm})$ & $161.5 \pm 8.4$ & $162.1 \pm 9.0$ & 0.07 & $161.6 \pm 8.2$ & $162.2 \pm 9.1$ & 0.06 \\
\hline Weight (kg) & $62.1 \pm 14.6$ & $63.1 \pm 10.9$ & 0.09 & $62.9 \pm 11.2$ & $63.0 \pm 10.9$ & 0.01 \\
\hline Never-smoker & $2,255(54.4)$ & $71(51.4)$ & 0.06 & $77(56.2)$ & $71(51.88)$ & 0.09 \\
\hline FVC (\%) & $102.9 \pm 19.8$ & $103.3 \pm 14.6$ & -0.04 & $103.9 \pm 14.0$ & $103.3 \pm 14.6$ & -0.04 \\
\hline $\mathrm{FEV}_{1}(\%)$ & $105.5 \pm 18.5$ & $106.7 \pm 19.3$ & 0.00 & $106.8 \pm 18.1$ & $106.8 \pm 19.3$ & 0.00 \\
\hline Size on CT scan $(\mathrm{cm})$ & $2.6 \pm 1.4$ & $2.7 \pm 1.5$ & 0.04 & $2.5 \pm 1.4$ & $2.6 \pm 1.5$ & 0.12 \\
\hline \multicolumn{7}{|l|}{ Comorbidities } \\
\hline Diabetes mellitus & $685(13.5)$ & $17(12.3)$ & -0.13 & $18(13.1)$ & $17(12.4)$ & -0.02 \\
\hline Renal insufficiency & $126(3.0)$ & $8(5.8)$ & 0.12 & $4(2.9)$ & $7(5.1)$ & 0.09 \\
\hline Pulmonary disease & $513(12.4)$ & $12(8.7)$ & -0.13 & $7(5.1)$ & $12(8.8)$ & 0.13 \\
\hline Cardiovascular disease & $1,597(38.5)$ & $46(33.3)$ & -0.11 & $44(48.9)$ & $46(51.1)$ & 0.03 \\
\hline Cancer history & 745 (18.0) & $26(18.8)$ & 0.02 & $27(19.7)$ & $26(19.0)$ & -0.02 \\
\hline Neoadjuvant treatment & $27(0.7)$ & $2(1.4)$ & 0.07 & $1(0.7)$ & $2(1.5)$ & 0.06 \\
\hline Clinical T1 (vs. >T2) & $2,619(63.2)$ & $85(61.6)$ & 0.03 & $97(70.8)$ & $85(62.0)$ & 0.18 \\
\hline Clinical N0 (vs. >N1) & 3,804 (91.8) & $123(89.1)$ & 0.08 & $129(94.2)$ & $123(89.8)$ & 0.14 \\
\hline \multicolumn{7}{|l|}{ Extent of resection } \\
\hline Segmentectomy & $501(12.1)$ & $20(14.5)$ & 0.04 & $26(19.0)$ & $20(14.6)$ & -0.10 \\
\hline Lobectomy & $3,619(87.3)$ & $117(84.7)$ & -0.07 & $111(80.2)$ & $116(85.2)$ & 0.10 \\
\hline Pneumonectomy & $25(0.6)$ & $1(0.7)$ & 0.01 & 0 & $1(0.7)$ & 0.09 \\
\hline Bronchoplasty or angioplasty & $74(1.8)$ & $9(6.5)$ & 0.19 & $7(5.1)$ & $9(6.6)$ & 0.06 \\
\hline Mediastinal LN dissection & $4,018(96.9)$ & $134(97.1)$ & 0.00 & $132(96.4)$ & $133(97.1)$ & -0.04 \\
\hline Adenocarcinoma & $3,433(82.8)$ & 109 (79.6) & 0.10 & 109 (79.6) & 109 (79.6) & 0.03 \\
\hline
\end{tabular}

Values are presented as mean \pm standard deviation or number $(\%)$, unless otherwise stated.

VATS, video-assisted thoracoscopic surgery; RATS, robot-assisted thoracoscopic surgery; SMD, standardized mean difference; FVC, forced vital capacity; $\mathrm{FEV}_{1}$, forced expiratory volume in one second; CT, computed tomography; LN, lymph node. 
Table 2. Perioperative data of the study population

\begin{tabular}{|c|c|c|c|c|c|c|}
\hline \multirow{2}{*}{ Variable } & \multicolumn{3}{|c|}{ Unmatched patients } & \multicolumn{3}{|c|}{ Propensity score-matched patients } \\
\hline & VATS $(\mathrm{N}=4,145)$ & RATS $(\mathrm{N}=138)$ & p-value & VATS $(\mathrm{N}=137)$ & RATS (N=137) & $\mathrm{p}$-value \\
\hline Thoracotomy conversion & $314(7.6)$ & $1(0.7)$ & 0.001 & $9(6.6)$ & $1(0.7)$ & 0.008 \\
\hline Hospital stay (day) & $5.9 \pm 6.0$ & $5.0 \pm 3.6$ & 0.087 & $5.8 \pm 3.9$ & $5.0 \pm 3.6$ & 0.052 \\
\hline Complication rate & 785 (18.9) & $26(18.8)$ & 0.829 & $24(17.5)$ & $26(19.0)$ & 0.874 \\
\hline$\geq$ Grade III & $227(5.5)$ & $7(5.1)$ & 1.000 & $6(4.4)$ & $7(5.1)$ & 1.000 \\
\hline 30-day mortality & $4(0.1)$ & 0 & 1.000 & 0 & 0 & - \\
\hline Complete resection & $4,125(99.5)$ & $136(98.6)$ & 0.157 & $134(97.8)$ & $135(98.5)$ & 1.000 \\
\hline No. of resected lymph nodes & $24.3 \pm 10.3$ & $24.1 \pm 8.8$ & 0.825 & $23.0 \pm 9.4$ & $24.0 \pm 8.8$ & 0.0370 \\
\hline Nodal upstaging & $475(11.5)$ & $24(17.4)$ & 0.033 & $13(9.5)$ & $24(17.5)$ & 0.052 \\
\hline cN0/pN1 & $212(5.1)$ & $12(8.7)$ & 0.6186 & $9(6.6)$ & $12(8.8)$ & 0.664 \\
\hline $\mathrm{cN} 0 / \mathrm{pN} 2$ & $260(6.3)$ & $12(8.7)$ & 0.915 & $4(2.9)$ & $12(8.8)$ & 0.077 \\
\hline
\end{tabular}

Values are presented as mean \pm standard deviation or number (\%), unless otherwise stated.

VATS, video-assisted thoracoscopic surgery; RATS, robot-assisted thoracoscopic surgery.

Table 3. Cause of conversion to thoracotomy

\begin{tabular}{lcc}
\hline \multirow{2}{*}{ Variable } & Propensity score-matched patients \\
\cline { 2 - 3 } & VATS $(\mathrm{N}=9)$ & RATS $(\mathrm{N}=1)$ \\
\hline Technical difficulty & 8 & 0 \\
Anthracotic lymph node & 3 & 0 \\
Extensive adhesion & 2 & 0 \\
Angioplasty & 2 & 0 \\
Bronchoplasty & 1 & 0 \\
Uncontrolled bleeding & 1 & 1 \\
\hline
\end{tabular}

VATS, video-assisted thoracoscopic surgery; RATS, robot-assisted thoracoscopic surgery.

cotomy because of technical difficulty including anthracofibrotic lymph nodes, extensive adhesion, bronchoplasty/ angioplasty, and uncontrolled bleeding (Table 3). One patient in the matched RATS group (0.7\%) required conversion to thoracotomy due to uncontrolled bleeding. The postoperative complication rate (VATS $17.5 \%$ versus RATS $19.0 \%, \mathrm{p}=0.874$ ) was not significantly different between the 2 groups. The length of hospital stay tended to be slightly shorter in the RATS group than in the VATS group (VATS $5.8 \pm 3.9$ days versus RATS $5.0 \pm 3.6$ days, $\mathrm{p}=0.052$ ). The complete resection rate (VATS $97.8 \%$ versus RATS $98.5 \%$, $\mathrm{p}=1.000$ ) and number of resected lymph nodes (VATS $23.0 \pm 9.4$ versus RATS $24.0 \pm 8.8, \mathrm{p}=0.370$ ) were similar between the 2 groups. In the unmatched cohort, nodal upstaging in the RATS group was significantly higher than in the VATS group (VATS $11.5 \%$ versus RATS $17.4 \%$, $\mathrm{p}=$ $0.033)$. However, the difference lost significance in the matched cohort (VATS 9.5\% versus RATS $17.5 \%, \mathrm{p}=0.052$ ). $\mathrm{N} 1$ nodal upstaging (cN0/pN1) was similar between the 2 groups (VATS 6.6\% versus RATS 8.8\%, $\mathrm{p}=0.664$ ). N2 nodal upstaging $(\mathrm{cN} 0 / \mathrm{pN} 2)$ tended to be more common in the
RATS group than in the VATS group, but without statistical significance (VATS $4 \%$ versus RATS $12 \%, \mathrm{p}=0.077$ ) in the matched cohort.

\section{Discussion}

We compared the early clinical outcomes of VATS and RATS for anatomical lung cancer surgery. Our propensity-matched analysis revealed that RATS was safe and had satisfactory short-term oncologic outcomes, in terms of postoperative complications and complete resection with sufficient lymph node yield. In addition, RATS was associated with a lower conversion rate than that of VATS despite technically demanding procedures, which were more commonly performed in RATS.

The previous literature consistently showed a lower conversion rate for RATS than for VATS $[15,16]$. Furthermore, the maximal utilization of robotic techniques makes it possible to perform sleeve resection with decreased bleeding loss, early tube removal, shorter operative time, and similar survival compared to VATS and thoracotomy [1719]. In experienced hands, intraoperative bleeding can be managed without conversion to thoracotomy [20]. In particular, anthracofibrotic lymph nodes are a significant predictor of intraoperative conversion to thoracotomy, as shown in our study [21]. In tuberculosis-endemic regions, hilar anthracofibrosis results in massive bleeding or injury to the bronchus while surgeons dissect the adjacent pulmonary vessels and bronchus. However, robotic techniques allow meticulous dissection between the pulmonary artery and bronchus at the level of the distal part, which makes the dissection safe.

The number of dissected lymph nodes and nodal upstag- 
ing are essential components of surgical resection for lung cancer. As part of oncologic clearance, nodal upstaging and the number of harvested lymph nodes are surrogates for the quality of lung cancer surgery. Our study showed that the number of resected lymph nodes was similar between RATS and VATS; however, nodal upstaging tended to be more common in the RATS group than in the VATS group. Previous studies have revealed that RATS achieved a higher lymph node yield and upstaging rate than those of VATS $[3,22]$, which might be associated with meticulous hilar and mediastinal dissection and the excellent surgical view offered by the robotic technique. Similarly, locally advanced cancers are potential candidates for the robotic technique [17-19].

VATS has been established as a standard minimally invasive approach for lung cancer. However, there have been discrepancies in the utilization of VATS according to region and hospital volume [23-25]. VATS or RATS is strongly recommended when there is no compromise of oncologic principles [26]. At experienced centers, minimally invasive surgery was found to improve early outcomes including reduced pain, decreased length of hospital stay, rapid functional recovery, and low complications with comparable oncologic outcomes in treating lung cancer. However, VATS anatomical resection had a significant learning curve [27, 28]. Meanwhile, RATS may flatten the learning curve of the surgical technique to gain the necessary proficiency without previous experience of minimally invasive surgery $[29,30]$. Consequently, the utilization of RATS for lung cancer has increased faster than that of VATS in the United States [23].

Some limitations of the present study should be noted. First, there might have been selection bias due to the retrospective nature of this study, which was conducted at a single institution, and the lack of randomization. Nonetheless, we conducted propensity score matching to minimize selection bias. Moreover, long-term clinical outcomes were not evaluated, warranting further research.

In conclusion, robotic anatomical resection for lung cancer resulted in comparable early outcomes compared with those of VATS. In particular, robotic resection resulted in a lower rate of conversion to thoracotomy. Furthermore, a robotic approach might improve lymph node harvesting in the N2 station.

\section{Conflict of interest}

No potential conflict of interest relevant to this article was reported.

\section{ORCID}

Ji Hyeon Park: https://orcid.org/0000-0002-3272-0067

Samina Park: https://orcid.org/0000-0001-9625-2672

Chang Hyun Kang: https://orcid.org/0000-0002-1612-1937

Bub Se Na: https://orcid.org/0000-0002-3293-1446

So Young Bae: https://orcid.org/0000-0003-3438-4973

Kwon Joong Na: https://orcid.org/0000-0003-4158-9790

Hyun Joo Lee: https://orcid.org/0000-0002-3092-2167

In Kyu Park: https://orcid.org/0000-0003-3550-5554

Young Tae Kim: https://orcid.org/0000-0001-9006-4881

\section{References}

1. Scott WJ, Allen MS, Darling G, et al. Video-assisted thoracic surgery versus open lobectomy for lung cancer: a secondary analysis of data from the American College of Surgeons Oncology Group Z0030 randomized clinical trial. J Thorac Cardiovasc Surg 2010;139:976-83.

2. Nwogu CE, D'Cunha J, Pang H, et al. VATS lobectomy has better perioperative outcomes than open lobectomy: CALGB 31001, an ancillary analysis of CALGB 140202 (Alliance). Ann Thorac Surg 2015;99:399-405.

3. Wilson JL, Louie BE, Cerfolio RJ, et al. The prevalence of nodal upstaging during robotic lung resection in early stage non-small cell lung cancer. Ann Thorac Surg 2014;97:1901-7.

4. Louie BE, Wilson JL, Kim S, et al. Comparison of video-assisted thoracoscopic surgery and robotic approaches for clinical stage I and stage II non-small cell lung cancer using The Society of Thoracic Surgeons Database. Ann Thorac Surg 2016;102:917-24.

5. Kneuertz PJ, D'Souza DM, Richardson M, Abdel-Rasoul M, Moffatt-Bruce SD, Merritt RE. Long-term oncologic outcomes after robotic lobectomy for early-stage non-small-cell lung cancer versus video-assisted thoracoscopic and open thoracotomy approach. Clin Lung Cancer 2020;21:214-24.

6. Liang H, Liang W, Zhao L, et al. Robotic versus video-assisted lobectomy/segmentectomy for lung cancer: a meta-analysis. Ann Surg 2018;268:254-9.

7. Toosi K, Velez-Cubian FO, Glover J, et al. Upstaging and survival after robotic-assisted thoracoscopic lobectomy for non-small cell lung cancer. Surgery 2016;160:1211-8.

8. Park BJ, Melfi F, Mussi A, et al. Robotic lobectomy for non-small cell lung cancer (NSCLC): long-term oncologic results. J Thorac Cardiovasc Surg 2012;143:383-9.

9. Huang J, Li C, Li H, et al. Robot-assisted thoracoscopic surgery versus thoracotomy for c-N2 stage NSCLC: short-term outcomes of a randomized trial. Transl Lung Cancer Res 2019;8:951-8.

10. Veronesi G, Park B, Cerfolio R, et al. Robotic resection of stage III lung cancer: an international retrospective study. Eur J Cardiothorac Surg 2018;54:912-9. 
11. Li C, Hu Y, Huang J, et al. Comparison of robotic-assisted lobectomy with video-assisted thoracic surgery for stage IIB-IIIA non-small cell lung cancer. Transl Lung Cancer Res 2019;8:820-8.

12. Cerfolio RJ. Total port approach for robotic lobectomy. Thorac Surg Clin 2014;24:151-6.

13. Pearlstein DP. Robotic lobectomy utilizing the robotic stapler. Ann Thorac Surg 2016;102:e591-3.

14. Kang $\mathrm{CH}$. The anterolateral approach in robotic lung cancer surgery. Ann Thorac Surg 2019;108:e401-3.

15. Andersson SE, Ilonen IK, Palli OH, Salo JA, Rasanen JV. Learning curve in robotic-assisted lobectomy for non-small cell lung cancer is not steep after experience in video-assisted lobectomy; single-surgeon experience using cumulative sum analysis. Cancer Treat Res Commun 2021;27:100362.

16. Reddy RM, Gorrepati ML, Oh DS, Mehendale S, Reed MF. Robotic-assisted versus thoracoscopic lobectomy outcomes from high-volume thoracic surgeons. Ann Thorac Surg 2018;106:902-8.

17. Cerfolio RJ. Robotic sleeve lobectomy: technical details and early results. J Thorac Dis 2016;8(Suppl 2):S223-6.

18. Qiu T, Zhao Y, Xuan Y, et al. Robotic sleeve lobectomy for centrally located non-small cell lung cancer: a propensity score-weighted comparison with thoracoscopic and open surgery. J Thorac Cardiovasc Surg 2020;160:838-46.

19. Lin MW, Kuo SW, Yang SM, Lee JM. Robotic-assisted thoracoscopic sleeve lobectomy for locally advanced lung cancer. J Thorac Dis 2016;8:1747-52

20. Cerfolio RJ, Bess KM, Wei B, Minnich DJ. Incidence, results, and our current intraoperative technique to control major vascular injuries during minimally invasive robotic thoracic surgery. Ann Thorac
Surg 2016;102:394-9.

21. Byun CS, Lee S, Kim DJ, et al. Analysis of unexpected conversion to thoracotomy during thoracoscopic lobectomy in lung cancer. Ann Thorac Surg 2015;100:968-73.

22. Zhang Y, Chen C, Hu J, et al. Early outcomes of robotic versus thoracoscopic segmentectomy for early-stage lung cancer: a multi-institutional propensity score-matched analysis. J Thorac Cardiovasc Surg 2020;160:1363-72.

23. Subramanian MP, Liu J, Chapman WC Jr, et al. Utilization trends, outcomes, and cost in minimally invasive lobectomy. Ann Thorac Surg 2019;108:1648-55.

24. Cooper MA, Hutfless S, Segev DL, Ibrahim A, Lyu H, Makary MA. Hospital level under-utilization of minimally invasive surgery in the United States: retrospective review. BMJ 2014;349:g4198.

25. Phillips JD, Bostock IC, Hasson RM, et al. National practice trends for the surgical management of lung cancer in the CMS population: an atlas of care. J Thorac Dis 2019;11(Suppl 4):S500-8.

26. National Comprehensive Cancer Network. Non-small cell lung cancer, 2021. Plymouth Meeting (PA): National Comprehensive Cancer Network; 2021.

27. Petersen RH, Hansen HJ. Learning curve associated with VATS lobectomy. Ann Cardiothorac Surg 2012;1:47-50.

28. McKenna RJ Jr. Complications and learning curves for video-assisted thoracic surgery lobectomy. Thorac Surg Clin 2008;18:275-80.

29. Arnold BN, Thomas DC, Bhatnagar V, et al. Defining the learning curve in robot-assisted thoracoscopic lobectomy. Surgery 2019;165: 450-4.

30. Hernandez JM, Humphries LA, Keeling WB, et al. Robotic lobectomy: flattening the learning curve. J Robot Surg 2012;6:41-5. 